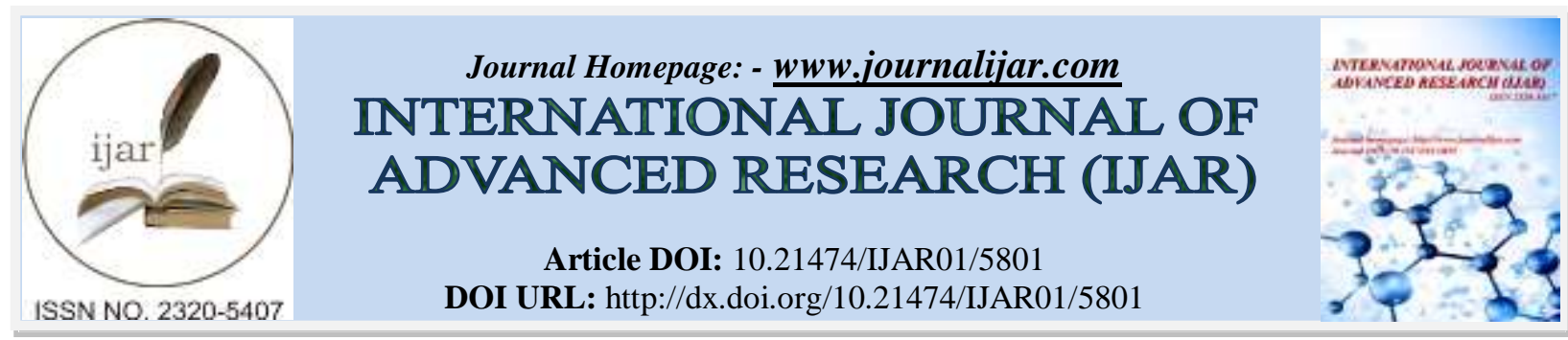

RESEARCH ARTICLE

\title{
THE EFFECT OF RADIOTHERAPY ON DENTAL PULP STATUS IN PATIENTS WITH HEAD AND NECK CANCER: A SYSTEMATIC REVIEW.
}

\author{
Mohamed Nageh ${ }^{1}$, Alaa ELBaz ${ }^{2}$ and Geraldine. Ahmed ${ }^{2}$. \\ 1. PhD student, Endodontic Department, Cairo University, Assistant Lecturer, Endodontic Department, Fayoum \\ University. \\ 2. Associate Professor, Endodontic Department, Cairo University.
}

\section{Manuscript Info}

Manuscript History

Received: 07 September 2017

Final Accepted: 09 October 2017

Published: November 2017

\begin{abstract}
Introduction: The purpose of this systematic review was to evaluate the literature and update our current understanding of the impact of present cancer radiotherapy (RT) on the dental pulp vitality. Method: A systematic literature search was conducted with assistance from a research librarian in the databases MEDLINE/PubMed, Cochrane, and Lilacs for published articles till May 2017. Each study was independently assessed by three reviewers. Taking into account recording the demographic data, tumor site, radiation dose, radiation device, follow up periods, method of pulp sensitivity testing, and collecting the results and conclusion of each article in tables, also critical appraisal of each article was performed using CASP checklist for case control and cohort studies. Results: Six eligible published papers between 1986 and 2016 were included and reviewed for a descriptive systematic review. Conclusion: The dental pulp in the pathway of ionizing radiation during RT of the head and neck region may not undergo either a complete loss or a decrease in vasculature and blood flow; therefore it may be prudent to wait at least for 5 months after RT to check for pulp vitality status. There continues to be lack of clinical studies on the effect of radiotherapy on the dental pulp health status especially on posterior teeth.
\end{abstract}

Copy Right, IJAR, 2017,. All rights reserved.

\section{Introduction:-}

Endodontics is the specialty of dentistry that deals with changes in the dental pulp and periradicular tissues and intended to evaluate the morphology, physiology and pathology. Study and practice in this area include the basic sciences including; biology of the normal pulp, the etiology, diagnosis, prevention and treatment of diseases and injuries that reach the pulp, associated or not to change perirradicular area (1). The diagnosis of endodontic change is one of most important steps for the correct treatment. The process begins from recording the past medical history of the patient, interpretation of the signs and symptoms, and testing of pulp vitality.

Some systemic disease may influence the dental treatment plan, such as patient receiving radiotherapy (RT) for the treatment of head and neck cancer; where prophylactic dental treatment as periodontal therapy, restoration of carious teeth, extraction of decayed teeth, root canal therapy of exposed teeth are needed before RT beginning; to avoid the 
development of osteoradionecrosis. Radiotherapy to the head and neck region causes xerostomia and salivary gland dysfunction which dramatically increases the risk of dental caries and its sequelae. Radiation therapy (RT) also affects the dental hard tissues increasing their susceptibility to demineralization following RT. Postradiation caries is a rapidly progressing and highly destructive type of dental caries. Radiation-related caries and other dental hard tissue changes can appear within the first 3 months following RT. There is a transient loss of pulp response to deep caries due to RT. Hence, every effort should be focused on prevention to manage patients with severe caries. This can be accomplished through good preoperative dental treatment, frequent dental evaluation and treatment after RT (with the exception of extractions), and consistent home care that includes self-applied fluoride.

During the medical planning of Head and neck radiotherapy, both tumor volume and normal anatomic structures of the maxillofacial region are included in the primary of radiation (2). Radiation therapy (RT) is part of the treatment of malignant tumors because of the ability of ionizing radiation to control cell growth. However, to reach target cells, radiation will have to pass through healthy tissues, which may damage the DNA of normal cells (3). Radiation side effects are mucositis, loss of taste, xerostomia, dental caries, fungal and bacterial infections, osteoradionecrosis, difficulty in speech and mastication, periodontal disease, fibrosis of soft tissues, trismus, endarteritis, hyperpigmentation of the skin surface in the field of radiation, transient hair loss, and loss of pulpal vitality. A decrease in pulp vascularity has been noted with radiotherapy (3-7). The determination of pulp vitality is an important step to assess the health or pathology of the pulp.

Clinical data suggest normal blood flow and sensitivity of dental pulp are impaired in such patients. However, little is known about the direct effects of radiation on pulp status, only a few previous studies attempted to investigate the direct effects of radiation on the dental pulp, some of which revealed significant loss of pulp vitality after RT (3, 57). Others revealed no significant loss of pulp vitality after RT $(4,8)$. Hence, there is great diversity of results when it comes to the ability of radiation to directly affect the dental pulp components and to further impair pulp vascularization and innervation, leading to clinically detectable teeth sensitivity changes to thermal and electrical stimulus, it also causes changes in the levels of oxygen saturation $(3,5-7)$. Considering the fact that responses of the dental pulp to thermal and electrical stimuli are directly related to the mechanisms of inflammation, microvascularization, and innervation of the dental pulp and little conflicting clinical data are available on pulp vitality after radiotherapy in head and neck malignant tumors patients; the current systematic review was performed. To our knowledge this is the first systematic review of clinical studies in the literature that evaluates the direct effects of RT on normal healthy tooth vitality or sensitivity, and to determine the time needed for the pulp tissues to regain its vitality.

\section{Objectives:-}

This systematic review aims to review all the available clinical studies that evaluated the effect of RT on the dental pulp status in healthy vital teeth of patients suffering from malignat tumors in the head and neck.

\section{Method:-}

\section{Search Strategy:-}

We searched the following electronic bibliographic databases: Pubmed, The Cochrane Library and Lilacs. In addition to hand searching of the identified journals' indexes, special issues, bibliographies and reference lists of identified articles were scanned to identify other potentially relevant articles. Structured electronic search was carried out including only terms relating to the intervention. The search terms are: Teeth pulp OR pulp vitality OR pulp sensitivity OR dental pulp AND radiation therapy OR radiation OR ionizing radiation exposure OR ionizing radiation OR radiation dose OR radiotherapy. The search was last updated on 30 May 2017. Papers in all languages are included. Criteria for including and excluding studies in the review are presented in table 1.

Table 1:- showing the inclusion and exclusion criteria for considering studies in the review based on the PICO structure.

\begin{tabular}{|l|l|}
\hline Inclusion Criteria & Exclusion Criteria \\
\hline 1- Clinical studies & 1-Animal studies \\
2-Head and neck cancer patients. & 2-Effect of radiotherapy on tooth ( enamel, dentin, \\
3-Adult patients. & cementum) structure \\
5-Utilizing the dental pulp sensitivity or vitality test. & 2-Effect of radiotherapy on tooth formation and \\
& development, and on periodontal status. \\
\hline
\end{tabular}


The results of the search strategy in Pubmed, Cochrane and Lilacs sources yielded 6 articles included after screening by title and abstract. Thus 6 full-text articles were assessed for eligibility. (Figure1)

Figure1:- Flow diagram of search results.

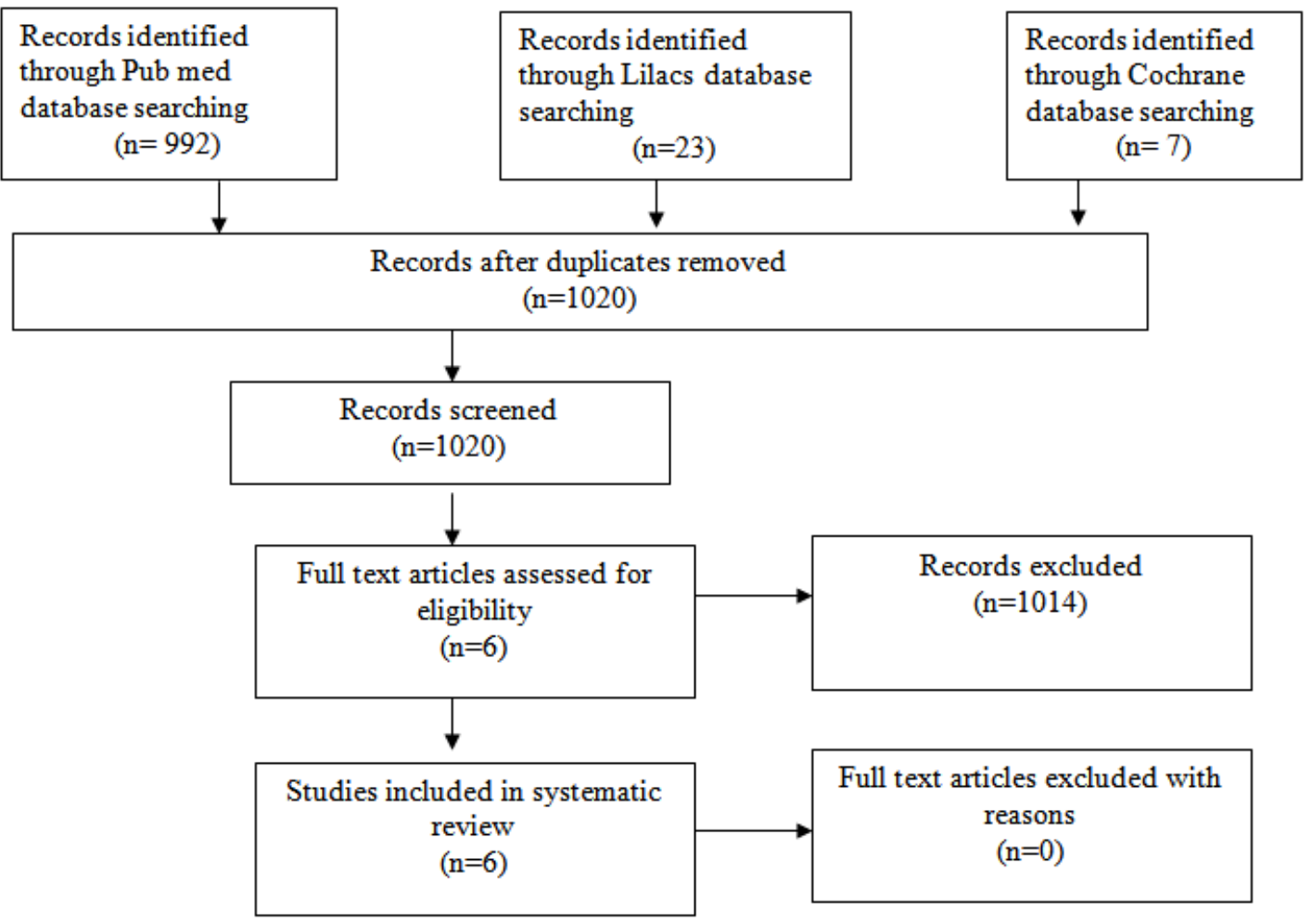

\section{Methods of Review:-}

Data extraction Strategy: Titles and abstracts of studies retrieved using the search strategy and those from additional sources were screened independently by the two review authors to identify studies that potentially meet the inclusion criteria outlined above. The full text of these potentially included studies were retrieved and independently assessed for eligibility by the two review team members.

A standardized, pre-piloted form used to extract data from the included studies for assessment of study quality and evidence synthesis. Extracted information include: demographic data, included teeth and tumor sites as shown in Table 2

\section{Critical Appraisal for the Included Studies:-}

A standardized assessment of methodological quality of each paper is carried out to improve inter and intra rater reliability by applying the critical appraisal approach used by CASP. A 10-question checklist was completed for each study http://www.casp-uk.net/casp-tools-checklists. Any disagreement was solved by discussion 
Table 2:- Demographic data

\begin{tabular}{|c|c|c|c|c|c|c|}
\hline Author & $\begin{array}{l}\text { Treated } \\
\text { teeth }\end{array}$ & Number & Age & Gender & Race & Tumor site \\
\hline $\begin{array}{l}\text { Knowles et } \\
\text { al } 1986(5)\end{array}$ & $\begin{array}{l}\text { Teeth in } \\
\text { maxilla } \\
\text { and } \\
\text { mandible }\end{array}$ & $\begin{array}{l}\text { Radiotherapy } \\
\text { group = 24 } \\
\text { patient ( } 389 \\
\text { teeth) } \\
\text { Control } \\
\text { group = (288 } \\
\text { teeth) }\end{array}$ & $\begin{array}{l}\text { Not } \\
\text { mentioned }\end{array}$ & $\begin{array}{l}\text { Not } \\
\text { mentioned }\end{array}$ & $\begin{array}{l}\text { Not } \\
\text { mentioned }\end{array}$ & $\begin{array}{l}\text { Oral and perioral } \\
\text { malignancies. }\end{array}$ \\
\hline $\begin{array}{l}\text { Rodrigues et } \\
\text { al } 2007 \text { (6) }\end{array}$ & $\begin{array}{l}\text { Lower } \\
\text { Incisors } \\
\text { and } \\
\text { Canines } \\
\text { Upper } \\
\text { Incisors } \\
\text { and } \\
\text { Canines }\end{array}$ & $\begin{array}{l}\text { Radiotherapy } \\
\text { group = } 12 \\
\text { patient }(91 \\
\text { teeth) } \\
\text { Control } \\
\text { group = } 12 \\
\text { patients (103 } \\
\text { teeth) }\end{array}$ & $\begin{array}{l}40-74 \text { years } \\
\text { The mean } \\
\text { age }=54.4 \\
\text { years }\end{array}$ & $\begin{array}{l}\text { Both } \\
\text { Male = } 16 \\
\text { Female = } \\
8\end{array}$ & Brazilian & $\begin{array}{l}10 \text { received radiotherapy for } \\
\text { treating squamous cell } \\
\text { carcinomas, one for melanoma } \\
\text { evil and one for medullary } \\
\text { thyroid carcinoma. The } \\
\text { localization tumors included } \\
\text { tonsil ( } 3 \text { patients), tongue } \\
\text { edge ( } 2 \text { patients), soft palate } \\
\text { (1 patient), and base of tongue } \\
\text { (1 patient), tongue belly ( } 1 \\
\text { patient), anterior pillar ( } 1 \\
\text { patient), thyroid ( } 1 \text { patient), } \\
\text { zygomatic area ( } 1 \text { patient) and } \\
\text { throat (1 patient). }\end{array}$ \\
\hline $\begin{array}{l}\text { Kataoka et al } \\
\text { 2011(4) }\end{array}$ & $\begin{array}{l}\text { Two } \\
\text { maxillary } \\
\text { or } \\
\text { mandibular } \\
\text { incisors of } \\
\text { each } \\
\text { patient }\end{array}$ & $\begin{array}{l}20 \text { pts } \\
\text { ( } 40 \text { teeth) }\end{array}$ & $\begin{array}{l}35 \text { to } 55 \\
\text { years }\end{array}$ & Both & Brazilian & $\begin{array}{l}\text { intraoral or } \\
\text { oropharyngeal malignant } \\
\text { tumors }\end{array}$ \\
\hline $\begin{array}{l}\text { Kataoka et al } \\
2012(3)\end{array}$ & $\begin{array}{l}\text { Two } \\
\text { maxillary } \\
\text { or } \\
\text { mandibular } \\
\text { incisors of } \\
\text { each } \\
\text { patient }\end{array}$ & $\begin{array}{l}20 \mathrm{pts} \\
\text { ( } 40 \text { teeth) }\end{array}$ & $\begin{array}{l}35 \text { to } 55 \\
\text { years } \\
\text { The mean } \\
\text { age }=47.2 \\
\text { years }\end{array}$ & Both & Brazilian & $\begin{array}{l}\text { intraoral or oropharyngeal } \\
\text { malignant tumors }\end{array}$ \\
\hline $\begin{array}{l}\text { Garg et al } \\
2015 \text { (7) }\end{array}$ & $\begin{array}{l}\text { Four } \\
\text { posterior } \\
\text { teeth, } 1 \\
\text { from } \\
\text { each } \\
\text { quadrant }\end{array}$ & $\begin{array}{l}\text { Twenty-one } \\
\text { patients } \\
\text { N teeth }=84\end{array}$ & $\begin{array}{l}40-65 \\
\text { years }\end{array}$ & $\begin{array}{l}\text { Not } \\
\text { mentioned }\end{array}$ & Indian & $\begin{array}{l}\text { malignant oral and } \\
\text { oropharyngeal }\end{array}$ \\
\hline $\begin{array}{l}\text { Kataoka et al } \\
2016(8)\end{array}$ & $\begin{array}{l}\text { lower } \\
\text { incisors } \\
\text { and } \\
\text { canines, } \\
\text { upper } \\
\text { incisors } \\
\text { and } \\
\text { canines }\end{array}$ & $\begin{array}{l}\text { Radiotherapy } \\
\text { group = 90 } \\
\text { patients ( } \\
693 \text { teeth) } \\
\text { Control } \\
\text { group = 90 } \\
\text { patients (693 } \\
\text { teeth) }\end{array}$ & $\begin{array}{l}\text { The mean } \\
\text { age of the } \\
\text { participants } \\
\text { in } \\
\text { radiotherapy } \\
\text { group was } \\
49.4 \text { years } \\
\text { (male, } 50.3 \\
\text { years; } \\
\text { female, } 48.5 \\
\text { years), }\end{array}$ & Both & Brazilian & $\begin{array}{l}\text { The most prevalent site of } \\
\text { head and neck cancer was the } \\
\text { oropharynx (66\%), followed } \\
\text { by the oral cavity (20\%), the } \\
\text { nasopharynx }(8 \%) \text {, and the } \\
\text { hypopharynx }(6 \%) \text {. }\end{array}$ \\
\hline
\end{tabular}




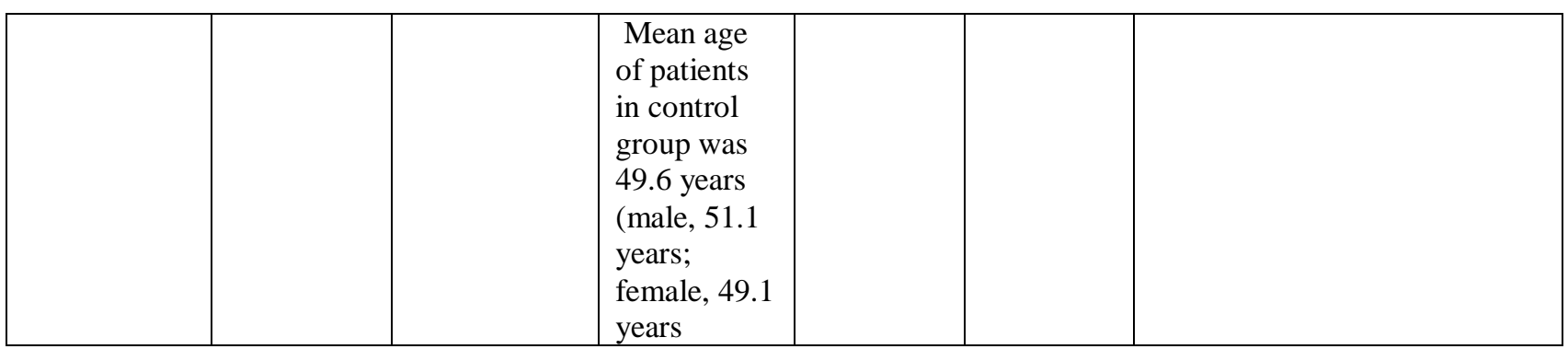

\section{Results:-}

The results of the systematic search included a net result of 6 articles to be included in the systematic review. Study design, radiotherapy dose, radiation device used, pulp sensitivity/vitality assessment device, follow up periods, results, and conclusion of each of the included studies are tabulated in table 3.

Table 3:- Results

\begin{tabular}{|c|c|c|c|c|c|c|c|c|}
\hline Author & $\begin{array}{l}\text { Study } \\
\text { design }\end{array}$ & Groups & $\begin{array}{l}\text { Dose } \\
\text { therapy }\end{array}$ & $\begin{array}{l}\text { Radia- } \\
\text { tion } \\
\text { device }\end{array}$ & $\begin{array}{l}\text { Pulp } \\
\text { sensiti- } \\
\text { vity } \\
\text { assess- } \\
\text { ment- } \\
\text { method }\end{array}$ & $\begin{array}{l}\text { Follow } \\
\text { - up } \\
\text { peri- } \\
\text { ods }\end{array}$ & Results & Conclusion \\
\hline \multirow[t]{3}{*}{$\begin{array}{l}\text { Knowle } \\
\text { s,et al } \\
1986(5)\end{array}$} & \multirow[t]{3}{*}{$\begin{array}{l}\text { Observ } \\
\text { ational } \\
\text { study }\end{array}$} & $\begin{array}{l}\text { Group } \\
\text { 1:Teeth } \\
\text { tested } \\
\text { before } \\
\text { RT }\end{array}$ & \multirow[t]{3}{*}{$\begin{array}{l}\text { minimal } \\
\text { dose of } \\
5000 \\
\text { rads } \\
\text { of cobalt- } \\
60 \\
\text { radiation } \\
\text { to the } \\
\text { head and } \\
\text { neck, }\end{array}$} & \multirow[t]{3}{*}{$\begin{array}{l}\text { Not } \\
\text { menti- } \\
\text { oned }\end{array}$} & \multirow[t]{3}{*}{$\begin{array}{l}\text { Electric } \\
\text { Pulp } \\
\text { tester }\end{array}$} & \multirow[t]{3}{*}{$\begin{array}{l}6 \\
\text { month } \\
\text { interva } \\
\text {-ls in a } \\
\text { 2-year } \\
\text { Period. }\end{array}$} & $\begin{array}{l}\text { mean decrease in } \\
\text { pulp sensitivity } \\
\text { Group 1: } \\
\text { Irradiated maxillary = } \\
\text { none } \\
\text { Irradiated mandibular } \\
=10.13 \\
\text { Non-irradiated } \\
\text { maxillary }=5.44 \\
\text { Non-irradiated } \\
\text { mandibular }=6.27 \\
\end{array}$ & \multirow{3}{*}{$\begin{array}{l}\text { Decreased } \\
\text { sensitivity was } \\
\text { observed in } \\
\text { teeth within } \\
\text { and adjacent to } \\
\text { an irradiated } \\
\text { field. } \\
\text { Mandibular } \\
\text { teeth outside } \\
\text { the field and } \\
\text { distal to the } \\
\text { irradiated } \\
\text { mandibular } \\
\text { nerve trunk } \\
\text { showed an } \\
\text { immediate } \\
\text { decrease in } \\
\text { sensitivity. } \\
\text { Maxillary } \\
\text { teeth outside } \\
\text { the field } \\
\text { showed a } \\
\text { delayed } \\
\text { decrease in } \\
\text { sensitivity. } \\
\text { Blood flow } \\
\text { rates and } \\
\text { nutrition were } \\
\text { also related to } \\
\text { time. } \\
\text { Neurons are } \\
\text { thought to be } \\
\text { relatively } \\
\end{array}$} \\
\hline & & $\begin{array}{l}\text { Group 2: } \\
\text { Teeth } \\
\text { first } \\
\text { tested } \\
\text { less than } \\
36 \\
\text { months } \\
\text { after RT }\end{array}$ & & & & & $\begin{array}{l}\text { Group } 2: \\
\text { Irradiated maxillary }= \\
16.13 \\
\text { Irradiated mandibular } \\
=14.90 \\
\text { Non-irradiated } \\
\text { maxillary }=6.14 \\
\text { Non-irradiated } \\
\text { mandibular }=6.02\end{array}$ & \\
\hline & & $\begin{array}{l}\text { Group 3: } \\
\text { Teeth } \\
\text { first } \\
\text { tested } \\
\text { more } \\
\text { than } 36 \\
\text { months } \\
\text { after RT }\end{array}$ & & & & & $\begin{array}{l}\text { Group 3: } \\
\text { Irradiated maxillary = } \\
14.00 \\
\text { Irradiated mandibular } \\
=0.90 \\
\text { Non-irradiated } \\
\text { maxillary = } 3.69 \\
\text { Non-irradiated }\end{array}$ & \\
\hline
\end{tabular}




\begin{tabular}{|c|c|c|c|c|c|c|c|c|}
\hline & & & & & & & mandibular $=3.45$ & radio-resistant \\
\hline & & $\begin{array}{l}\text { Group 4: } \\
\text { Teeth } \\
\text { non- } \\
\text { irradiated } \\
\text { (control } \\
\text { patients) }\end{array}$ & & & & & $\begin{array}{l}\text { Group 4: } \\
\text { Irradiated maxillary }= \\
0.61 \\
\text { Irradiated mandibular } \\
=0.42 \\
\text { Non-irradiated } \\
\text { maxillary }=0.61 \\
\text { Non-irradiated } \\
\text { mandibular }=0.42\end{array}$ & $\begin{array}{l}\text { seen } \\
\text { histologically } \\
\text { after radiation } \\
\text { therapy. } \\
\text { However, } \\
\text { functional } \\
\text { impairment } \\
\text { was observed } \\
\text { in } \\
\text { histologically } \\
\text { normal tissue. }\end{array}$ \\
\hline \multirow[t]{3}{*}{$\begin{array}{l}\text { Rodrigu } \\
\text { es et al } \\
2007 \text { (6) }\end{array}$} & \multirow[t]{3}{*}{$\begin{array}{l}\text { Observ } \\
\text { ational } \\
\text { study }\end{array}$} & $\begin{array}{l}\text { Group 1: } \\
\text { Teeth } \\
\text { irradiated }\end{array}$ & \multirow{3}{*}{$\begin{array}{l}\text { The } \\
\text { radiation } \\
\text { doses } \\
\text { varied } \\
\text { from } \\
1400- \\
7120 \\
\text { cGy, } \\
\text { accordin- } \\
\text { g to the } \\
\text { applicati- } \\
\text { on areas } \\
\text { (tumor } \\
\text { primary, } \\
\text { clavicula } \\
\text {-r fossa, } \\
\text { neck and } \\
\text { face), } \\
\text { and all } \\
\text { patients } \\
\text { received } \\
\text { at least } \\
4500 \\
\text { cGy at } \\
\text { some of } \\
\text { the sites } \\
\text { cited }\end{array}$} & \multirow[t]{3}{*}{$\begin{array}{l}\text { Not } \\
\text { menti- } \\
\text { oned }\end{array}$} & \multirow[t]{3}{*}{$\begin{array}{l}\text { Refrigera } \\
\text { nt } \\
\text { tetrafluor } \\
\text { o-ethane } \\
\text { gas }\end{array}$} & \multirow{3}{*}{$\begin{array}{l}\text { Mean } \\
\text { follow- } \\
\text { up } \\
\text { period } \\
\text { after } \\
4.3 \\
\text { months } \\
\text { of } \\
\text { radio- } \\
\text { therap } \\
\text { y }\end{array}$} & $\begin{array}{l}\text { The number of teeth } \\
\text { (percentage )of pulp } \\
\text { response } \\
\text { Group 1: } \\
\text { Positive = } 92(89.3 \\
\% \text { ) } \\
\text { Negative = } 11(10.7 \\
\%)\end{array}$ & \multirow{3}{*}{$\begin{array}{l}\text { Patients } \\
\text { undergoing } \\
\text { radiotherapy } \\
\text { for head and } \\
\text { neck had the } \\
\text { highest } \\
\text { number of } \\
\text { negative } \\
\text { responses to } \\
\text { the pulp } \\
\text { vitality test } \\
\text { using the } \\
\text { refrigerant } \\
\text { tetrafluoroetha } \\
\text { ne gas in } \\
\text { comparison } \\
\text { with the } \\
\text { control group }\end{array}$} \\
\hline & & $\begin{array}{l}\text { Group 2: } \\
\text { Teeth } \\
\text { non- } \\
\text { irradiated } \\
\text { (control } \\
\text { patients } \\
\end{array}$ & & & & & $\begin{array}{l}\text { Group 2: } \\
\text { Positive }=65(71.4 \\
\%) \\
\text { Negative }=26(28.6 \\
\%)\end{array}$ & \\
\hline & & & & & & & & \\
\hline \multirow[t]{2}{*}{$\begin{array}{l}\text { Kataoka } \\
\text { et al } \\
2011 \text { (4) }\end{array}$} & \multirow[t]{2}{*}{$\begin{array}{l}\text { Observ } \\
\text { ational } \\
\text { study }\end{array}$} & $\begin{array}{l}\text { Group 1: } \\
\text { (TP1), } \\
\text { before } \\
\text { Radiothe } \\
\text { rapy(RT) }\end{array}$ & \multirow{2}{*}{$\begin{array}{l}\text { radiation } \\
\text { doses } \\
\text { between } \\
30 \text { and } \\
35 \text { Gy; } \\
\text { TP3, at } \\
\text { the end } \\
\text { of RT } \\
\text { with } \\
\text { radiation } \\
\text { doses } \\
\text { between } \\
60 \text { and } \\
70 \text { Gy }\end{array}$} & \multirow[t]{2}{*}{$\begin{array}{l}\text { Not } \\
\text { mentio } \\
\text { ned }\end{array}$} & \multirow[t]{2}{*}{$\begin{array}{l}\text { Pulse } \\
\text { oximetry }\end{array}$} & \multirow{2}{*}{$\begin{array}{l}\text { Before } \\
\text { RT, at } \\
\text { begin- } \\
\text { ing of } \\
\text { RT, at } \\
\text { the } \\
\text { end of } \\
\text { RT, 4- } \\
5 \\
\text { months } \\
\text { after } \\
\text { RT }\end{array}$} & $\begin{array}{l}\text { Mean \% of pulp } \\
\text { oxygenation level } \\
(\mathrm{SpO} 2) \\
\text { Group } 1(\mathrm{TP} 1)= \\
93 \%\end{array}$ & \multirow{2}{*}{$\begin{array}{l}\text { Because the } \\
\text { mean \%SpO2 } \\
\text { before RT was } \\
\text { greater than } \\
\text { during and } \\
\text { after therapy } \\
\text { and values } \\
\text { obtained } 4 \text { to } 5 \\
\text { months after } \\
\text { the beginning } \\
\text { of RT were } \\
\text { close to the } \\
\text { initiation of } \\
\text { RT, pulp tissue }\end{array}$} \\
\hline & & $\begin{array}{l}\text { Group 2: } \\
(\mathrm{TP} 2) \text { at } \\
\text { the } \\
\text { beginnin } \\
\mathrm{g} \text { of } \mathrm{RT} \\
\text { with } \\
\text { radiation }\end{array}$ & & & & & $\begin{array}{l}\text { Group } 2(\mathrm{TP} 2)= \\
83 \%\end{array}$ & \\
\hline
\end{tabular}




\begin{tabular}{|c|c|c|c|c|c|c|c|c|}
\hline & & $\begin{array}{l}\text { dose } \\
\text { between } \\
30 \text { and } \\
35 \mathrm{~Gy}\end{array}$ & & & & & $77 \%$ & $\begin{array}{l}\text { may be able to } \\
\text { regain normal } \\
\text { blood flow } \\
\text { after RT. }\end{array}$ \\
\hline & & $\begin{array}{l}\text { Group 3: } \\
\text { ( TP3), at } \\
\text { the end } \\
\text { of RT } \\
\text { with } \\
\text { radiation } \\
\text { doses } \\
\text { between } \\
60 \text { and } \\
70 \text { Gy }\end{array}$ & & & & & $\begin{array}{l}\text { Group } 4(\mathrm{TP} 4)= \\
85 \%\end{array}$ & $\begin{array}{l}\text { If the changes } \\
\text { in the } \\
\text { microcirculati } \\
\text { on of the } \\
\text { dental pulp } \\
\text { were indeed } \\
\text { transitory, } \\
\text { preventive } \\
\text { endodontic }\end{array}$ \\
\hline & & $\begin{array}{l}\text { Group 4: } \\
\text { (TP4 ), } 4 \\
\text { to } 5 \\
\text { months } \\
\text { after the } \\
\text { beginnin } \\
\text { g of } \\
\text { cancer } \\
\text { treatment }\end{array}$ & & & & & & $\begin{array}{l}\text { treatment or } \\
\text { extraction in } \\
\text { patients who } \\
\text { are currently } \\
\text { undergoing or } \\
\text { recently } \\
\text { received RT } \\
\text { and who show } \\
\text { negative signs } \\
\text { of pulp } \\
\text { sensitivity may } \\
\text { not be } \\
\text { necessary for } \\
\text { pulpal reasons. }\end{array}$ \\
\hline $\begin{array}{l}\text { Kataoka } \\
\text { et al } \\
2012 \text { (3) }\end{array}$ & $\begin{array}{l}\text { Observ } \\
\text { ational } \\
\text { study }\end{array}$ & $\begin{array}{l}\text { Group 1: } \\
\text { (TP1), } \\
\text { before } \\
\text { Radiothe } \\
\text { rapy(RT) }\end{array}$ & $\begin{array}{l}\text { radiation } \\
\text { doses } \\
\text { between } \\
30 \quad \text { and } \\
35 \quad \text { Gy; }\end{array}$ & $\begin{array}{l}\text { 3- } \\
\text { dimens } \\
\text { ional } \\
\text { confor } \\
\text { mal }\end{array}$ & $\begin{array}{l}\text { cold } \\
\text { thermal } \\
\text { pulp } \\
\text { sensitivit } \\
\text { y testing }\end{array}$ & $\begin{array}{l}\text { Before } \\
\text { RT, at } \\
\text { beginn } \\
\text { ing of } \\
\text { RT, at }\end{array}$ & $\begin{array}{l}\text { Mean \% of pulpal } \\
\text { response to (PST) } \\
\text { Group } 1(\mathrm{TP} 1)= \\
100 \%\end{array}$ & $\begin{array}{l}\text { RT decreased } \\
\text { the number of } \\
\text { teeth } \\
\text { responding to } \\
\text { PST after }\end{array}$ \\
\hline & & $\begin{array}{l}\text { Group 2: } \\
\text { (TP2) at } \\
\text { the } \\
\text { beginnin } \\
\mathrm{g} \text { of } \mathrm{RT}\end{array}$ & $\begin{array}{l}\text { TP3, at } \\
\text { the end } \\
\text { of RT } \\
\text { with } \\
\text { radiation }\end{array}$ & $\begin{array}{l}\text { radioth } \\
\text { erapy } \\
\text { (3D- } \\
\text { RT) or } \\
\text { Intensi }\end{array}$ & (PST) & $\begin{array}{l}\text { end of } \\
\text { RT, } 4- \\
5 \\
\text { months } \\
\text { after }\end{array}$ & $\begin{array}{l}\text { Group } 2(\mathrm{TP} 2)= \\
22.5 \% \\
(18.8 \%) \text { in the 3D-RT } \\
(25.0 \%) \text { in the IMRT }\end{array}$ & $\begin{array}{l}\text { doses greater } \\
\text { than } 30 \text { to } 35 \\
\text { Gy. } \\
\text { The type of } \\
\text { RT (3D-RT or }\end{array}$ \\
\hline & & $\begin{array}{l}\text { with } \\
\text { radiation }\end{array}$ & & $\begin{array}{l}\text { ty- } \\
\text { modul }\end{array}$ & & RT & Group $3(\mathrm{TP} 3)=0 \%$ & $\begin{array}{l}\text { IMRT) had no } \\
\text { influence on }\end{array}$ \\
\hline & & $\begin{array}{l}\text { dose } \\
\text { between } \\
30 \text { and } \\
35 \mathrm{~Gy}\end{array}$ & $\begin{array}{l}60 \text { and } \\
70 \mathrm{~Gy}\end{array}$ & $\begin{array}{l}\text { ated } \\
\text { radioth } \\
\text { erapy } \\
\text { (IMRT }\end{array}$ & & & Group $4(\mathrm{TP} 4)=0 \%$ & $\begin{array}{l}\text { the pulp } \\
\text { responses to } \\
\text { PST after the } \\
\text { conclusion of }\end{array}$ \\
\hline & & $\begin{array}{l}\text { Group 3: } \\
\text { ( TP3), at } \\
\text { the end } \\
\text { of RT } \\
\text { with } \\
\text { radiation } \\
\text { doses } \\
\text { between } \\
60 \text { and } \\
70 \mathrm{~Gy}\end{array}$ & & & & & & RT \\
\hline & & $\begin{array}{l}\text { Group 4: } \\
\text { (TP4 ), } 4\end{array}$ & & & & & & \\
\hline
\end{tabular}




\begin{tabular}{|c|c|c|c|c|c|c|c|c|c|c|c|}
\hline & & $\begin{array}{l}\text { to } 5 \\
\text { months } \\
\text { after the } \\
\text { beginnin } \\
\text { of } \\
\text { treatment }\end{array}$ & & & & & & & & & \\
\hline \multirow[t]{16}{*}{$\begin{array}{l}\text { Garg et } \\
\text { al } 2015 \\
\text { (7) }\end{array}$} & \multirow[t]{16}{*}{$\begin{array}{l}\text { Observ } \\
\text { ational } \\
\text { study }\end{array}$} & $\begin{array}{l}\text { Group 1: } \\
\text { in time } \\
\text { before } \\
\text { radiother } \\
\text { apy } \\
\text { (PT1) }\end{array}$ & \multirow{16}{*}{$\begin{array}{l}\text { radiation } \\
\text { doses } \\
\text { between } \\
30 \text { and } \\
35 \text { Gy; } \\
\text { TP3, at } \\
\text { the end } \\
\text { of RT } \\
\text { with } \\
\text { radiation } \\
\text { doses } \\
\text { between } \\
66 \text { and } \\
70 \text { Gy }\end{array}$} & \multirow[t]{16}{*}{$\begin{array}{l}\text { Intensi } \\
\text { ty- } \\
\text { modul } \\
\text { ated } \\
\text { radioth } \\
\text { erapy }\end{array}$} & \multirow{16}{*}{$\begin{array}{l}\text { Cold } \\
\text { thermal } \\
\text { pulp } \\
\text { sensitivit } \\
\text { y testing } \\
\text { ( PST) } \\
\text { and } \\
\text { electric } \\
\text { pulp } \\
\text { testing } \\
\text { (EPT) }\end{array}$} & \multirow{16}{*}{$\begin{array}{l}\text { Before } \\
\text { RT, at } \\
\text { PT2, } \\
\text { PT3, } \\
\text { end of } \\
\text { RT, } 4 \\
\text { months } \\
\text { from } \\
\text { beginn } \\
\text { ing of } \\
\text { RT }\end{array}$} & \multicolumn{4}{|c|}{$\begin{array}{l}\text { Mean of pulpal } \\
\text { response to (EPT) in } \\
1 \text { st quadrant }\end{array}$} & \multirow{16}{*}{$\begin{array}{l}\text { Radiotherapy } \\
\text { decreased the } \\
\text { number } \\
\text { of teeth } \\
\text { responding to } \\
\text { pulp } \\
\text { sensitivity } \\
\text { testing after } \\
\text { doses greater } \\
\text { than } 30-35 \mathrm{~Gy}\end{array}$} \\
\hline & & $\begin{array}{l}\text { Group 2: } \\
\text { after } 30-\end{array}$ & & & & & $\begin{array}{l}\text { PT } \\
1\end{array}$ & \begin{tabular}{|l|} 
PT \\
2 \\
\end{tabular} & \begin{tabular}{|l|} 
PT \\
3 \\
\end{tabular} & $\begin{array}{l}\mathrm{PT} \\
4\end{array}$ & \\
\hline & & $\begin{array}{l}35 \text { Gy } \\
(\text { PT2) }\end{array}$ & & & & & $\begin{array}{l}13 \\
.8\end{array}$ & $\begin{array}{l}16 . \\
71\end{array}$ & $\begin{array}{l}21 . \\
00\end{array}$ & $\begin{array}{l}36 . \\
19\end{array}$ & \\
\hline & & Group 3: & & & & & & & & & \\
\hline & & $\begin{array}{l}\text { at the } \\
\text { end of } \\
\text { radiother } \\
\text { apy at }\end{array}$ & & & & & \multicolumn{4}{|c|}{$\begin{array}{l}\text { Mean of pulpal } \\
\text { response to (EPT) in } \\
2 \text { nd quadrant }\end{array}$} & \\
\hline & & $\begin{array}{l}66-70 \\
\text { Gy (PT3) }\end{array}$ & & & & & $\begin{array}{l}\text { PT } \\
1\end{array}$ & $\begin{array}{l}\text { PT } \\
2\end{array}$ & $\begin{array}{l}\text { PT } \\
3\end{array}$ & $\begin{array}{l}\text { PT } \\
4\end{array}$ & \\
\hline & & $\begin{array}{l}\text { Group 4: } \\
4 \text { months } \\
\text { after } \\
\text { beginnin }\end{array}$ & & & & & $\begin{array}{l}12 \\
.5 \\
2\end{array}$ & $\begin{array}{l}14 . \\
81\end{array}$ & $\begin{array}{l}19 . \\
62\end{array}$ & $\begin{array}{l}30 . \\
76\end{array}$ & \\
\hline & & $\begin{array}{l}\mathrm{g} \text { of } \\
\text { radiother } \\
\text { apy } \\
\text { (PT4) }\end{array}$ & & & & & \multicolumn{4}{|c|}{$\begin{array}{l}\text { Mean of pulpal } \\
\text { response to (EPT) in } \\
3 \text { rd quadrant }\end{array}$} & \\
\hline & & & & & & & $\begin{array}{l}\text { PT } \\
1\end{array}$ & \begin{tabular}{|l}
$\mathrm{PT}$ \\
2
\end{tabular} & $\begin{array}{l}\text { PT } \\
3\end{array}$ & $\begin{array}{l}\text { PT } \\
4\end{array}$ & \\
\hline & & & & & & & $\begin{array}{l}14 \\
.0 \\
5\end{array}$ & $\begin{array}{l}15 . \\
81\end{array}$ & $\begin{array}{l}18 . \\
86\end{array}$ & $\begin{array}{l}31 . \\
00\end{array}$ & \\
\hline & & & & & & & \multicolumn{4}{|c|}{$\begin{array}{l}\text { Mean of pulpal } \\
\text { response to (EPT) in } \\
4 \text { th quadrant }\end{array}$} & \\
\hline & & & & & & & $\begin{array}{l}\text { PT } \\
1 \\
\end{array}$ & \begin{tabular}{|l|}
$\mathrm{PT}$ \\
2 \\
\end{tabular} & \begin{tabular}{|l|}
$\mathrm{PT}$ \\
3 \\
\end{tabular} & $\begin{array}{l}\mathrm{PT} \\
4 \\
\end{array}$ & \\
\hline & & & & & & & $\begin{array}{l}13 \\
.7 \\
6\end{array}$ & $\begin{array}{l}17 . \\
38\end{array}$ & $\begin{array}{l}20 . \\
10\end{array}$ & $\begin{array}{l}33 . \\
05\end{array}$ & \\
\hline & & & & & & & \multicolumn{4}{|c|}{$\begin{array}{l}\text { Mean \% of pulpal } \\
\text { response to (PST) in } \\
1 \text { st quadrant }\end{array}$} & \\
\hline & & & & & & & $\begin{array}{l}\text { PT } \\
1 \\
\end{array}$ & \begin{tabular}{|l|} 
PT \\
2 \\
\end{tabular} & $\begin{array}{l}\text { PT } \\
3 \\
\end{array}$ & $\begin{array}{l}\text { PT } \\
4\end{array}$ & \\
\hline & & & & & & & 10 & 23. & $0 \%$ & 0 & \\
\hline
\end{tabular}




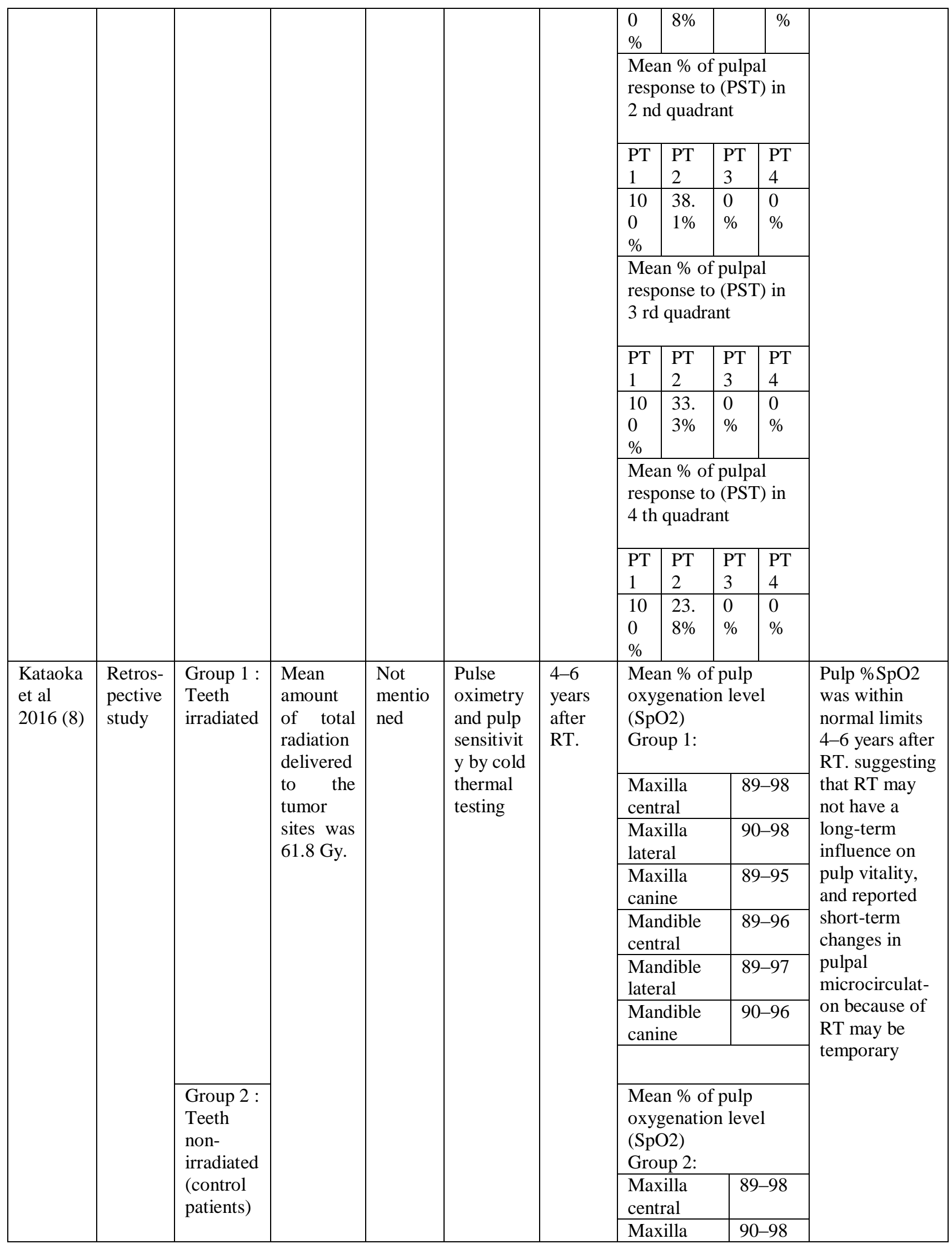




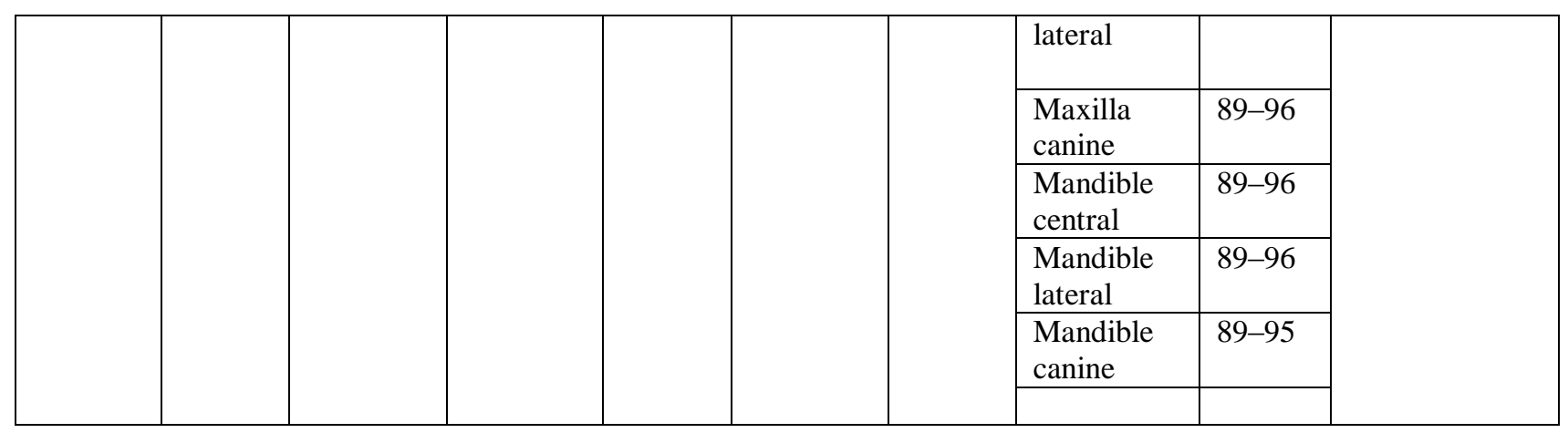

\section{Discussion:-}

We are providing a narrative synthesis of the findings from the 6 included studies, structured around same PICO; P: permanent vital teeth. I: patients exposed to radiotherapy for treatment of head and neck tumors. C: patients not exposed to radiotherapy. O: retaining pulp vitality. The available clinical cases all are published in form of case series, case control, and cohort studies. There is not any randomized controlled trial in this field. In the hierarchy of evidence, cohort studies represents level III evidence, case series represents level IV evidence (9) but they are the core in the review because they were the only available in-vivo clinical data.

The included studies were carried out on patients who were post-head and neck RT, likely because these individuals are thought to be at a much higher risk for the development of dental complications (10). The 6 included papers summed 2421 teeth; with 1337 teeth exposed to radiation dose during treatment of head and neck malignant tumors with RT, and 1084 teeth act as control in patients not exposed to RT. In the included articles, the age of the patients were from 40-74 years old without mentioning the prevalence of patient's sex in 4 studies, one study (7) didn't mention the sex included in the study, only one study (6) showed the prevalence of male patients. The races of population in this study were in Indian (7) and Brazilian people $(3,4,6,8)$. Thus, that finding recommends doing clinical studies on more races of population.

Apparently, patients undergoing RT may exhibit a negative response to pulp sensitivity tests (7). This phenomenon could be caused by the fact that patients affected by radiation-related caries do not present dental pain on a routine basis. Several hypotheses suggest that the direct effects of radiation on the dental pulp would be able to negatively affect the metabolism of odontoblasts and compromise the response of the dentin-pulp complex to the progression of radiation related caries, explaining the absence of pain even in deep carious lesions $(3,11)$. However, other studies do not support direct radiation damage to the innervation of the pulp that would be able to negatively affect the viability of odontoblasts, the repair capacity of dentin against caries progression, and the inhibition of pulp sensitivity and responses to caries progression $(10,12,13)$. As the result of this controversial scenario, it is sometimes stated anecdotally that radiogenic destruction of collagen within the dental pulp contributes to fibrosis and decreased vascularity and sensitivity (14). However, there are few data in the literature to confirm or refuse this claim. The diagnosis of pulp vitality is highly important because changes can develop into processes that may involve the periradicular tissues and predispose patients to developing osteoradionecrosis (4). Thus the present systematic review was performed to evaluate clinically the effect of radiation dose on dental pulp status.

The results from the collected data confirmed that there is a transient decrease in the pulp response to sensitivity and vitality tests, this decrease lasts from a period of 4- 5 months after healthy teeth exposure to radiotherapy. Also results showed that RT decreased the number of teeth responding to pulp sensitivity or vitality testing after doses greater than 30-35 Gy. Also one study done by Kataoka et al 2016 (8) found that percent of pulp oxygenation level to be within normal limits 4-6 years after RT. This suggests that RT may not have a long-term influence on pulp vitality, and reported short-term changes in pulpal microcirculation because of RT may be temporary. But only one study by Garg et al 2015(7) showed that no tooth (0\%) responded to pulp sensitivity testing after 4-5 months of RT. It must be put in mind that this study ( 7) was performed only on maxillay and posterior teeth posterior teeth, while the other studies $(3,4,6,8)$ included only maxillary and mandibular anterior teeth, and one study (5) included maxillary and mandibular teeth without mentioning the type. The difference in the results of the study done by Garg et al 2015 (7) and the other studies $(3,4,6,8)$ could be explained due to the difference in the type of teeth included; The enamel and dentin thickness and number of roots differ between the anterior and posterior teeth (15), which 
could affect the pulp response to sensitivity tests (16), also the radiation exposure affect on the pulp differs according to the teeth type. Thus it is advised to perform more studies especially on the posterior teeth, since only one study was performed.

The explanation of this transient loss of pulp sensitivity or vitality may be due to the congestion of blood vessels during radiotherapy which can lead to ischemia, resulting in decreased or absent blood flow. The decrease in blood flow and subsequent hypoxia and anoxia can cause an inhibition of myelinic fibers $(17,18)$ and may lead to an immediate reduction of tooth sensitivity even in teeth outside the field of radiation(5). from the collected data, it was revealed that the increase in radiation dose above 30-35 Gy caused decrease in tooth sensitivity, which could be explained by Fajardo et al 2001 (19) who stated that acute hematologic abnormalities and thrombosis in the microcirculation start occurring at $1 \mathrm{~Gy}$ radiation. Also ionizing radiation causes chemical injuries in the tissues, and the radiation therapy may be the reason for changes in microcirculation (20). Also Kataoka et al 2011 (4) stated that irradiation of 2 Gy significantly destroyed small capillaries with diameters below $10 \mathrm{~nm}$. Larger capillaries and venules with diameters above $10 \mathrm{~nm}$ were impaired after the application of doses up to $20 \mathrm{~Gy}$. Therefore, changes in the microcirculation of the pulp could very well take place as early as in the beginning of RT with radiation doses between 30 and $35 \mathrm{~Gy}$ and then be more clearly noticeable by reduced pulp response with radiation doses between 60 and 70 Gy.

Supporting our review result that loss of pulpal response is transient and that no pulp necrosis occurs; Knowles et al 1986 (5) concluded that neurons are thought to be relatively radio-resistant and few changes were seen histologically after RT. Also Farira et al 2014 ( 21 ) concluded histologically, that the morphologic features of the dental pulp of patients who have undergone RT in the head and neck region were highly preserved, and no changes were found in the immunohistochemical expression of any of the proteins studied, suggesting preservation of the activity of the microvasculature, neural components, and extracellular matrix fibroblasts of the pulp after HNR.

In the last decade, a lot of attention has been given to improve irradiation treatment for tumors in the head and neck region. The aim of these improvements is to increase the therapeutic results and to reduce the negative side effects, due to the irradiation of the surrounding oral tissues and organs (22). Two types of devices are used in radiotherapy of head and neck malifnant tumors: 3-dimensional conformal radiotherapy (3D-RT) or Intensity-modulated radiotherapy (IMRT). The head and neck region is a well-suited location for the treatment of cancer with IMRT because of the complex anatomic topography of this area and the potential severity of radiation-associated tissue defects in the proximity of vital organs (23). A characteristic benefit of IMRT is that clinical targets are treated at a therapeutically highly effective dosage while healthy neighboring structures receive the maximum protection attainable (24) although it requires considerably more time than 3D-RT for treatment planning and execution (25). Several studies suggested that patients treated with IMRT suffer from less radiation-induced xerostomia (26-28) and thus provide patients with a major improvement in the quality of life (29). Merely even with this IMRT irradiation method, some of the teeth are still in the targeted area and alterations of hard dental tissue and mechanical properties cannot be prevented. In the literature, only one study by Kataoka et al 2012 (3) concluded that the type of RT (3DRT or IMRT) had no influence on the pulp responses to PST after finishing of RT.

The systematic review results showed that after a period from 4-5 months $(4,6)$ and from 4-6 years ( 8$)$, the teeth regained its pulpal response to sensitivity and vitality testing, which could be because of the vascular characteristics of the dental pulp; the possibility for remodeling or even formation of new vessels is not remote and points to a possible vascular recovery after RT. This is also based on the observation that viable arteriogenesis and vasculogenesis occur after dental trauma by the formation of new vessels in a low oxygen environment, with vascular endothelial growth factor as the most important cytokine for the process (29-32). Low oxygen levels may stimulate angiogenesis both in healthy tissues and in tumors. Rabbani et al (33) represented the hypothesis that tissue hypoxia serves as a trigger for hypoxia-inducible factor-1a activity. Once activated, this cytokine induces vascular endothelial growth factor expression, which is necessary to start and continue angiogenesis (34). Responses of the dental pulp to RT are directly related to the understanding of molecular mechanisms involved in the inflammatory, ischemic, and hypoxic processes that affect not only normal tissues but also tumors.

\section{Conclusion:-}

The systematic review concluded that more studies are needed especially on the posterior teeth, and on different races of population. Also the findings suggest that the dental pulp in the pathway of ionizing radiation during RT of the head and neck region may not undergo either a complete loss or a decrease in vasculature and blood flow, 
therefore it may be prudent to wait at least for 5 months after RT to check for pulp vitality status. The clinical changes commonly observed in the pulpal microcirculation, which were demonstrated to occur in the short-term, may only be of a temporary nature. This may suggest that often recommended preventive endodontic treatments or tooth extractions in patients who will receive RT could be prevented on a larger scale. Additionally, the risk of rampant tooth decay with its sudden onset and osteoradionecrosis is a lifelong threat. Thus, the diagnosis of pulp vitality is highly important in patients with malignant oral and oropharyngeal tumors undergoing RT because changes can involve periradicular tissues and predispose patients to developing osteoradionecrosis.

\section{Acknowledgments:-}

The authors deny any conflicts of interest related to this study

\section{Refrences:-}

1. Walton RE, Torabinejad M. Principles and Practice of Endodontic 2nd ed. Philadelphia: WB Saunders; 1994.

2. Hansen HJ,MaritimB, Bohle GC 3rd, et al. Dosimetric distribution to the tooth-bearing regions of the mandible following intensity-modulated: radiation therapy for base of tongue cancer. Oral Surg Oral Med Oral Pathol Oral Radiol 2012; 114:504.

3. Kataoka SH, Setzer FC, Fregnani ER, et al. Effects of 3-dimensional conformal or intensity-modulated radiotherapy on dental pulp sensitivity during and after the treatment of oral or oropharyngeal malignancies. $\mathbf{J}$ Endod 2012; 38:148-52.

4. Kataoka SH, Setzer FC, Gondim-Junior E. Pulp vitality in patients with intraoral and oropharyngeal malignant tumors undergoing radiation therapy assessed by pulse oximetry. J Endod 2011; 37:1197-2000.

5. Knowles JC, Chalian VA, Shidnia H. Pulp innervation after radiation therapy. J Prosthet Dent. 1986 Dec; 56(6):708-11.

6. Rodrigues HM, and Franzi SA. Evaluation of pulp response in patients undergone radiation therapy for treatment of head and neck cancer. Rev. Bras. Cir. Cabeça Pescoço, v. 36, nº 1, p. 23 - 26, janeiro / fevereiro / março 2007.

7. Garg H, Grewal MS, Rawat S, MD, Suhag A, Sood P B, Grewal S, BDS, and Ahlawat P. Dental Pulp Status of Posterior Teeth in Patients with Oral and Oropharyngeal Cancer Treated with Concurrent Chemoradiotherapy. J Endod. 2015 Nov;41(11):1830-3.

8. Kataoka SH, Setzer FC, Gondim-Junior E, Fregnani ER, Moraes CJ, Pessoa OF, Gavini G, Caldeira CL. Late Effects of Head and Neck Radiotherapy on Pulp Vitality Assessed by Pulse Oximetry. J Endod. 2016 Jun;42(6):886-9

9. Degen RM, Hodgins JL, Bhandari M. The language of evidence based medicine: answers to common questions? Indian J. Orthopaedics. 2008; 42(2):111-7.

10. Catherine H. L., Hong, JN, Brian D., Hodgson, Monique et al. A systematic review of dental disease in patients undergoing cancer therapy. Support Care Cancer (2010) 18:1007-1021.

11. Silva AR, Alves FA, Antunes A, et al. Patterns of demineralization and dentin reactions in radiation-related caries. Caries Res 2009; 43:43-9.

12. Silva AR, Alves FA, Berger SB, et al. Radiation-related caries and early restoration failure in head and neck cancer patients. A polarized light microscopy and scanning electron microscopy study. Support Care Cancer 2010; 18:83-7.

13. Kielbassa AM, Hinkelbein W, Hellwig E, et al. Radiation-related damage to dentition. Lancet Oncol 2006; 7:326-35.

14. Springer IN, Niehoff P, Warnke PH, et al. Radiation caries-radiogenic destruction of dental collagen. Oral Oncol 2005; 41:723-8.

15. Stroud JL, English J, Buschang PH. Enamel thickness of the posterior dentition: its implications for nonextraction treatment. Angle Orthod. 1998 Apr;68(2):141-6

16. Levin LG. Pulp and Periradicular Testing. J Endod 2013;39:S13-S19

17. Kim S. Neurovascular interactions in the dental pulp in health and inflammation. J Endod 1990;16:48-53.

18. Ahlquist M, Franzen O. Pulpal ischemia in man: effects on detection threshold, A-delta neural response and sharp dental pain. Endod Dent Traumatol 1999;15: 6-16.

19. Fajardo LP, Berthrong M, Anderson RE. Radiation Pathology. New York: Oxford University Press; 2001.

20. Caldeira CL, Fidel SR, Pesce HF, Aun CE. Avaliacao da respostapulparaos testes de vitalidade com frioem dentes com deposicao de dentinareparativa. RPG Revista de Pos-graduacao (USP) 1995;2:157-60. 
21. Faria KM, Brandão TB, Ribeiro AC, Vasconcellos AF, de Carvalho IT, de Arruda FF, Castro Junior G, Gross VC1, Almeida OP, Lopes MA, Santos-Silva AR. Micromorphology of the dental pulp is highly preserved in cancer patients who underwent head and neck radiotherapy. J Endod. 2014 Oct;40(10):1553-9.

22. Lieshout HF, Bots CP. The effect of radiotherapy on dental hard tissue--a systematic review. Clin Oral Investig. 2014 Jan; 18(1):17-24.

23. Bucci MK, Bevan A, Roach M III. Advances in radiation therapy: conventional to 3D, to IMRT, to 4D, and beyond. CA Cancer J Clin 2005; 55:117-34.

24. Luxton G, Hancock SL, Boyer AL. Dosimetry and radiobiologic model comparasion of IMRT and 3D conformal radiotherapy in treatment of carcinoma of the prostate. In J Radiat Oncol Biol Phys 2004; 59:267-84.

25. Rades D, Fehlauer F, Wroblesky J, Albers D, Schid SE, Schmidt R. Prognostic factors in head-and-neck cancer patients treated with surgery followed by intensitymodulated radiotherapy (IMRT), 3D-conformal radiotherapy, or conventional radiotherapy. Oral Oncol 2007;43:535-43.

26. Chao KSC, Ozyigit G, Tran BN, Cengiz M, Dempsey JF, Low DA. Patterns of failure in patients receiving definitive and postoperative IMRT for head-and-neck cancer. Int J Radiat Oncol Biol Phys 2003;55:312-21.

27. Rusthoven KE, Raben D, Ballonoff A, Kane M, Song JI, Chen C. Effect of radiation techniques in treatment of oropharynx cancer Laryngoscope 2008;118: 635-9.

28. Lin A, Kim HM, Terrell JE, Dawson LA, Eisbruch A. Quality of life after parotidsparing IMRT for head-andneck cancer: a prospective longitudinal study. Int J Radiat Oncol Biol Phys 2003; 57:61-70.

29. Lin S, Roguin A, Metzger Z, Levin L. Vascular endothelial growth factor (VEGF) response to dental trauma: a preliminary study in rats. Dent Traumatol 2008;24: 435-8.

30. Matuella LG, Figueiredo JAP, N€or JE, et al. Vascular endothelial growth factor receptor-2 expression in the pulp of human primary and young permanent teeth. J Endod 2007;33:1408-12.

31. Artese L, Rubini C, Ferrero G, et al. Vascular endothelial growth factor (VEGF) expression in health and inflamed human dental pulp. J Endod 2002;28: 20-3.

32. Sunderkotter C, Steinbrink K, Goebeler M, et al. Macrophages and angiogenesis. J Leukoc Biol 1994;55:41022

33. Rabbani ZN, Mi J, Zhang Y, et al. Hypoxia inducible factor 1 alpha signaling in fractionated radiation-induced lung injury: role of oxidative stress and tissue hypoxia. Radiat Res 2010;173:165-74.

34. Semenza GL. Hypoxia, clonal selection, and the role of HIF-1a in tumor progression. Crit Rev Biochem Mol Biol 2000;35:71-103. 